\title{
Impact of the COVID-19 pandemic on infectious disease hospitalizations of neonates at a tertiary academic hospital: a cross-sectional study
}

\author{
Jiarong Pan, Canyang Zhan, Tianming Yuan", Yi Sun, Weiyan Wang and Lihua Chen
}

\begin{abstract}
Background: To investigate the impact of the coronavirus disease 2019 (COVID-19) pandemic on hospitalizations for neonatal infectious diseases.

Methods: We analyzed data for neonatal inpatients admitted at a tertiary academic hospital with a principal diagnosis of an infectious disease during January 2015 to December 2020. We compared hospitalizations in 2020 (COVID19 cohort), corresponding with the impact of COVID-19 pandemic and associated containment measures, and the comparable 2015 to 2019 (pre-COVID-19 cohort).

Results: 14,468 cases admitted for neonatal infectious diseases were included in our study, with 1201 cases in the COVID-19 cohort and 13,267 cases in the pre-COVID-19 cohort. The leading causes of hospitalizations for neonatal infectious diseases remain being respiratory tract infections (median ratio $=0.461,95 \% \mathrm{Cl} 0.335-0.551$ ), sepsis (median ratio $=0.292,95 \% \mathrm{Cl} 0.263-0.361$ ), gastric intestinal infections (median ratio $=0.095,95 \% \mathrm{Cl} 0.078-0.118$ ) and dermatologic infections (median ratio $=0.058,95 \% \mathrm{Cl} 0.047-0.083$ ). The seasonality of neonatal infectious disease hospitalizations could be obviously observed, with the total number and the overall rate of hospitalizations for neonatal infectious diseases in the first and fourth quarters greater than that of hospitalizations for neonatal infectious diseases in the second and third quarters in each year (1362.67 \pm 360.54 vs $1048.67 \pm 279.23, P=0.001 ; 8176 / 20020$ vs $6292 / 19369, P<0.001$, respectively). Both the numbers and the proportions of hospitalizations for neonatal infectious diseases in different quarters of the COVID-19 cohort significantly decreased as compared with those forecasted with the data from the pre-COVID-19 cohort: the numbers per quarter ( $300.25 \pm 57.33$ vs $546.64 \pm 100.43$, P-value $=0.006$ ), the first quarter ( 0.34 vs $0.40, P=0.002)$, the second quarter $(0.24$ vs $0.30, P=0.001)$, the third quarter $(0.24$ vs 0.28 , $\mathrm{P}=0.024)$, and the fourth quarter $(0.29$ vs $0.35, P=0.003)$.
\end{abstract}

Conclusions: Despite the outbreak of the COVID-19 pandemic, the leading causes of hospitalizations for neonatal infectious diseases remain unchanged. The seasonality of neonatal infectious disease hospitalizations could be obviously observed. The numbers as well as the overall rates of hospitalizations for neonatal infectious diseases in the COVID-19 cohort dramatically declined with the impact of the COVID-19 pandemic and its mitigation measures.

\footnotetext{
*Correspondence: yuantianming@zju.edu.cn

Department of Neonatology, The Children's Hospital, Zhejiang University

School of Medicine, National Clinical Research Center for Child Health,

3333 Binsheng Road, Hangzhou 310052, Zhejiang, People's Republic

of China
}

(c) The Author(s) 2022. Open Access This article is licensed under a Creative Commons Attribution 4.0 International License, which permits use, sharing, adaptation, distribution and reproduction in any medium or format, as long as you give appropriate credit to the original author(s) and the source, provide a link to the Creative Commons licence, and indicate if changes were made. The images or other third party material in this article are included in the article's Creative Commons licence, unless indicated otherwise in a credit line to the material. If material is not included in the article's Creative Commons licence and your intended use is not permitted by statutory regulation or exceeds the permitted use, you will need to obtain permission directly from the copyright holder. To view a copy of this licence, visit http://creativecommons.org/licenses/by/4.0/. The Creative Commons Public Domain Dedication waiver (http://creativeco mmons.org/publicdomain/zero/1.0/) applies to the data made available in this article, unless otherwise stated in a credit line to the data. 
Keywords: Infection, Neonatology, Inpatient, COVID-19, Epidemiology

\section{Background}

Infectious diseases have traditionally been a major cause of morbidity and mortality in neonates [1-3], constituting one of the highest proportions of hospitalization during neonatal period [4]. Acquiring a better understanding of neonatal infectious diseases is crucial for decision making on public health priorities and appropriate management providing for newborn infants. Thus, it is very important to study the status and trends of infectious diseases in neonates.

The coronavirus disease 2019 (COVID-19) global pandemic is caused by a novel coronavirus named severe acute respiratory syndrome coronavirus 2 (SARS-CoV-2), which was first identified in December 2019 [5]. In 2020, as the COVID-19 rapidly developed into a global health emergency, national and local governments around the world introduced mandated lockdowns, quarantines, isolation, mask wearing, social distancing, and other measures to curb the pandemic in the interest of public health [6-8]. Although China has effectively reversed the pandemic situation and curbed the spread of COVID-19 in the first quarter of 2020 [9], great efforts including mask wearing, social distancing, vaccinations, test and trace strategies continue to struggle against COVID-19 [1012]. Not only have these mitigation measures effectively maintained containment of COVID-19, but also they have impacted the prevalence of hospitalizations for neonatal infectious diseases.

The aims of the present study were to analyze the trend of hospitalizations for neonatal infectious diseases at a tertiary academic hospital and to assess impact of COVID-19 pandemic and associated prevention and control measures on infectious disease hospitalizations of neonates.

\section{Methods}

\section{Study design and patients' data}

A hospital based cross-sectional study was conducted by collecting epidemiological data on hospital admissions for infectious diseases in neonatal inpatients at a tertiary academic hospital (the Children's Hospital, Zhejiang University School of Medicine) from January 2015 to December 2020. The age of patients who required hospitalization with principal diagnoses of infectious diseases in the data set ranged from 0 to 28 days. Our institution is a national clinical research center for child health and a tertiary hospital of pediatric healthcare that has approximately 3.5 million outpatient visits and 81,000 inpatient admissions every year. The prevalence of infectious disease hospitalizations of neonates has been impacted by COVID-19 pandemic and corresponding containment measures implemented since January 2020.

\section{Protocol}

The study protocol was approved by the ethics committee of the Children's Hospital, Zhejiang University School of Medicine (IRB/EC Ref No. 2021-IRB-166). All of the clinical data included in this study were fully anonymized and could no longer be retraced. The need for individual informed consent was waived by the ethics committee because of the retrospective nature and anonymity of the study.

\section{Inclusion criteria}

Included were all neonatal inpatients with: (i) admission age ranged from 0 to 28 days AND (ii) principal diagnoses of infectious diseases AND (iii) who were admitted from January 2015 to December 2020.

\section{Exclusion criteria}

Excluded were neonatal inpatients with: (i) repetitive records OR (ii) lack of medical information (eg, length of hospital stay $\leq 2$ days) OR (iii) non-infectious cases of newborn.

The variables collected for analysis included date of admission, date of discharge, length of stay, age, gender and principal diagnosis. An early newborn is defined as a newborn with postnatal age of less than 7 days and a late newborn is defined as a neonate with postnatal age of beyond 7 days. The diagnoses were made according to the International Classification of Diseases, version 10 (ICD-10). To facilitate the effective analysis, the diagnoses were grouped by using the subcategories criteria into respiratory infection, digestive system infection, urinary tract infection, intracranial infection, systemic infection and other or not specified infection. The cases with infection of two or more subcategories were coded with the first principal diagnoses. Clinical data were collected to compare hospitalizations during four quarters of 2020 (COVID-19 cohort), corresponding with the impact of COVID-19 pandemic and associated containment measures, and the comparable 2015 to 2019 quarters (preCOVID-19 cohort).

\section{Statistical analysis}

The categorical variables were described as absolute and relative frequencies, and numerical variables were described as mean values and standard deviations 
(SD). Categorical variables were compared between the COVID-19 and pre-COVID-19 cohorts by means of Chi-square tests. $T$-test was applied to compare normally distributed continuous variables. The simple seasonal model and Winters' additive model were set up to forecast the numbers of hospitalizations for neonatal infectious diseases and proportions of those to the total

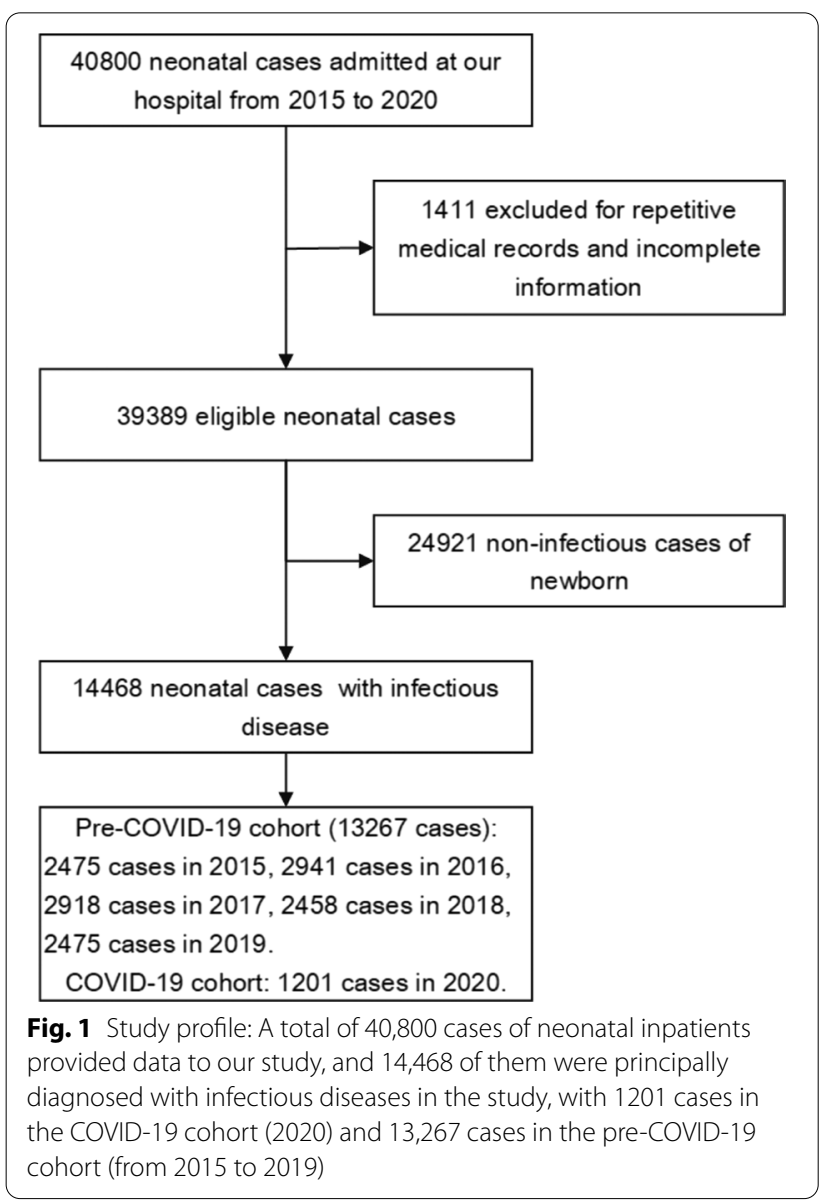

hospitalizations of neonates, respectively. All analyses were performed with SPSS Statistics for Windows (software version 26.0 ). All $P$ values $<0.05$ were considered statistically significant.

\section{Results}

Figure 1 illustrates study participant selection and distribution in different years. Of the 40,800 cases of neonatal inpatients providing data to our study, 39,389 met inclusion criteria and contributed patient data to the study. 14,468 of them were principally diagnosed with infectious diseases (An Additional file 1 shows this in more detail), corresponding to a mean neonatal infectious disease hospitalization rate of $36.73 \%$. There were 1201 cases in the COVID-19 cohort and 13,267 cases in the pre-COVID-19 cohort. All neonatal inpatients have received a nucleic acid test before admission in an effort to curb the spread of COVID-19 and ensure the safety of medical care in our hospital from January to December 2020, and all the results have been negative.

\section{Infectious disease hospitalizations of neonates by demographic group}

Categorization of ICD-10 codes for neonatal infectious disease cases by site of infection or sepsis is shown in Table 1. The demographic and clinical characteristics as well as the principal diagnoses are summarized in Table 2. This table also shows the comparisons of neonatal cases admitted for infectious diseases between the COVID-19 and pre-COVID-19 cohorts. The numbers of hospitalizations for neonatal infectious diseases of early newborns and late newborns in different quarters are shown in Fig. 2. In the hospitalizations for neonatal infectious diseases, late newborns are in the majority (64.8-87.8\%). As shown in Fig. 2, the trend of the number of hospitalizations for infectious diseases of early newborns is basically similar to that of late newborns,

Table 1 Categorization of ICD-10 codes for infectious diseases by site of infection or sepsis used to evaluate infectious disease hospitalizations of neonates, the Children's Hospital, Zhejiang University School of Medicine, 2015-2020

\begin{tabular}{ll}
\hline Category & ICD-10 code \\
\hline Respiratory tract infection & A37.0, A37.9, A49.3, B08.5, B97.4, J00.x, J02.9, J04.0, J04.2, J06.9, J10.0, J10.1, J11.0, J11.1, J12.1, J12.2, J16.0, J16.8, J18.0, \\
& J18.9, J20.4, J20.9, J40.x, P22.2, P22.3, P23.0, P23.1, P23.6, P23.8, P23.9, P28.8, P35.0, P39.9, R05.x \\
Sepsis & A41.2, A41.9, A50.9, B00.9, B01.9, B08.4, B25.8, B25.9, B34.0, B34.9, P35.1, P36.3, P36.4, P36.9, P37.1, P58.2, R50.9, R65.2, \\
& R65.3 \\
Gastric intestinal infection & A02.0, A04.9, A08.0, A08.4, A09.0, A09.9, K12.1, K35.0, K51.9, K52.9, P59.2, P77.X, R56.8 \\
Dermatologic infection & B09.X, B35.0, H60.9, H61.9, K61.0, L00.X, L01.0, L02.2, L02.8, L02.9, L03.1, L03.3, L03.8, L03.9, L08.0, L08.9, L98.9, P15.8, \\
& P36.2, P38.X, P39.4, R22.9 \\
Central nervous system infection & A39.0, A85.0, A86.X, A87.9, B00.3, B00.4, G00.8, G00.9, G03.9, G04.8, G06.0 \\
Urinary tract infection & N34.2, N39.0, P39.3, R82.7 \\
Other or not specified & A54.3, H00.0, H04.3, H10.9, H66.0, H66.4, H66.9, H70.9, I40.0, K11.2, K65.9, M00.9, N61.X, P39.0, P39.1, P39.8, P81.9, P83.4 \\
\hline
\end{tabular}


Table 2 Infectious disease hospitalizations of neonates by demographic group, the Children's Hospital, Zhejiang University School of Medicine, 2015-2020 $(n=14,468)$

\begin{tabular}{|c|c|c|c|}
\hline Total $(14,468)$ & Pre-COVID-19 cohort $(n=13,267)$ & COVID-19 cohort $(n=1201)$ & P-value \\
\hline Gender (male/female) & $7468 / 5799$ & $674 / 527$ & 0.909 \\
\hline Postnatal age (days) & $14.59 \pm 8.44$ & $15.30 \pm 8.12$ & 0.004 \\
\hline Age group (early newborn/late newborn) & $3041 / 10226$ & $214 / 987$ & $<0.001$ \\
\hline \multicolumn{4}{|c|}{ Classification of diagnostic subcategories for infectious disease } \\
\hline Respiratory infection & $6251(47.12 \%)$ & $556(46.29 \%)$ & 0.587 \\
\hline Systemic infection & $4390(33.09 \%)$ & $286(23.81 \%)$ & 0.000 \\
\hline Digestive system infection & $1255(9.46 \%)$ & $145(12.07 \%)$ & 0.004 \\
\hline Skin infection & $773(5.83 \%)$ & $96(7.99 \%)$ & 0.003 \\
\hline Intracranial infection & $271(2.04 \%)$ & $44(3.66 \%)$ & 0.000 \\
\hline Urinary tract infection & $106(0.80 \%)$ & $24(2.00 \%)$ & 0.000 \\
\hline Other or not specified infection & $221(1.67 \%)$ & $50(4.16 \%)$ & 0.000 \\
\hline Length of hospital stay (days) & $8.87 \pm 7.60$ & $8.65 \pm 7.44$ & 0.330 \\
\hline
\end{tabular}

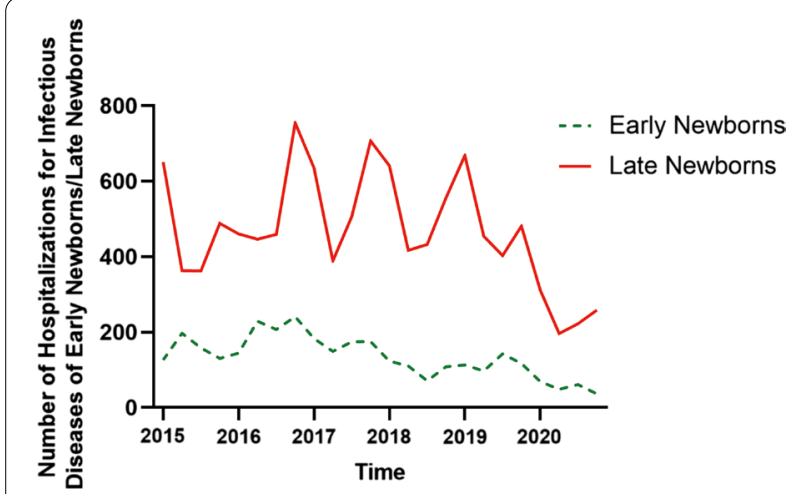

Fig. 2 The numbers of hospitalizations for neonatal infectious diseases of early and late newborns. The numbers of both early and late newborns impacted by the COVID-19 pandemic significantly decreased in different quarters of 2020. Q: quarter

and the numbers of quarterly hospitalizations for infectious diseases of both early newborns and late newborns were significantly lower in the COVID-19 cohort compared with the pre-COVID-19 cohort: early newborns ( $53.50 \pm 14.53$ vs $149.70 \pm 8.31, P=0.003$ ), late newborns (246.75 \pm 49.81 vs $513.65 \pm 104.98, P=0.003)$.

\section{Leading causes among infectious disease hospitalizations of neonates}

By analyzing the principal diagnoses of the data, it is found that there are many different causes of neonatal infectious diseases, with some of the most common ones being respiratory tract infections, sepsis, gastric intestinal infections, dermatologic infections, central nervous system infections and urinary tract infections (Fig. 3). With the proportions of causes changing in different quarters, the leading causes of hospitalizations for neonatal infectious diseases remain being respiratory tract infections (median ratio $=0.461$, 95\% CI $0.335-0.551$ ), sepsis (median ratio $=0.292,95 \%$ CI $0.263-0.361$ ), gastric intestinal infections (median ratio $=0.095,95 \%$ CI 0.078 0.118 ) and dermatologic infections (median ratio $=0.058$, $95 \%$ CI 0.047-0.083) in our study.

\section{Infectious disease hospitalizations of neonates by the seasonality}

The observed numbers and the rates of hospitalizations for neonatal infectious diseases fluctuated in different quarters from January 2015 to December 2020 (Fig. 4). The total number or the overall rate of hospitalizations for neonatal infectious diseases in the first and fourth quarters was greater than that of hospitalizations for neonatal infectious diseases in the second and third quarters in each year $(1362.67 \pm 360.54$ vs $1048.67 \pm 279.23$, $P=0.001 ; 8176 / 20020$ vs $6292 / 19369, P<0.001$, respectively). Obviously, the seasonality of neonatal infectious disease hospitalizations could be observed. As it fluctuated in different quarters, the seasonality of respiratory tract infectious disease hospitalizations could also be obviously observed, with the total number or the overall rate in the first and fourth quarters greater than that in the other two quarters in our study $(767.50 \pm 197.78$ vs $367.00 \pm 109.00, P<0.001 ; 4605 / 20020$ vs $2202 / 19369$, $P<0.001$, respectively) (Fig. 4).

\section{Infectious disease hospitalizations of neonates by the impact of the COVID-19 pandemic}

The simple seasonal model was applied to forecast the numbers of hospitalizations for neonatal infectious diseases in different quarters of 2020 with the data in the pre-COVID-19 cohort. As shown in Fig. 5a, the numbers of hospitalizations for neonatal infectious 


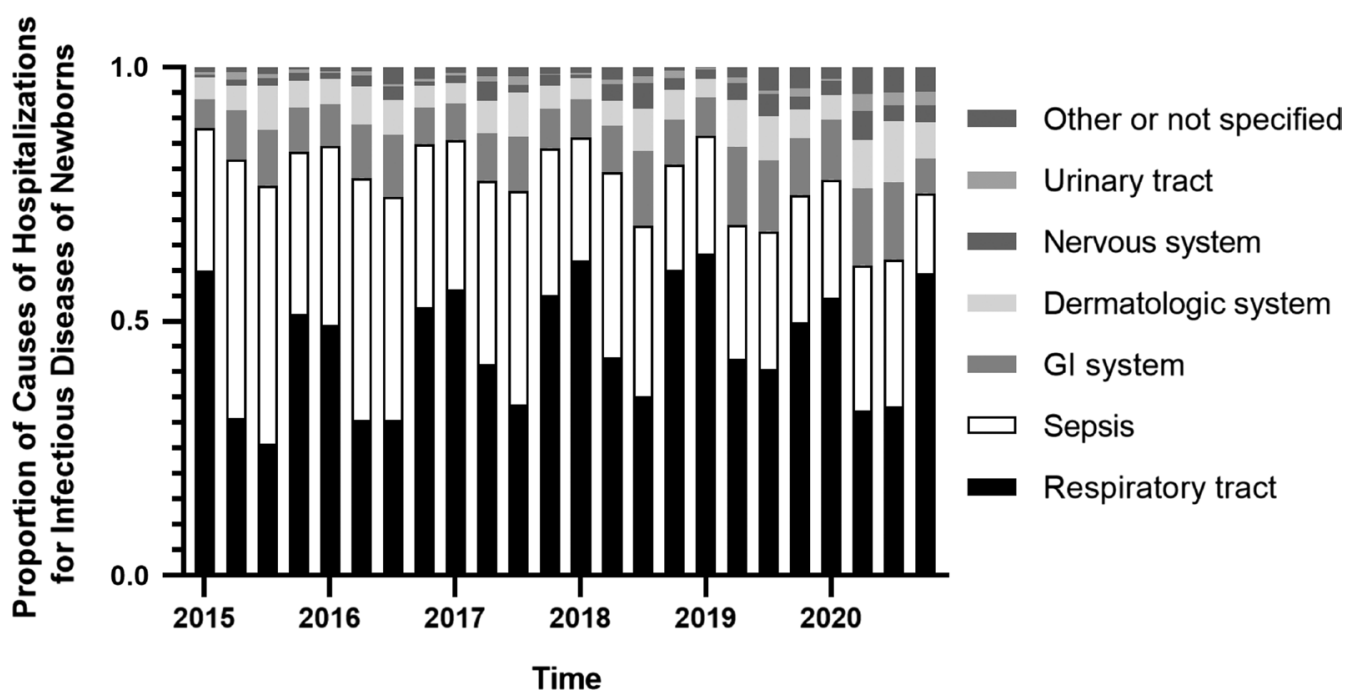

Fig. 3 The proportions of causes of hospitalizations for neonatal infectious diseases. The leading causes of hospitalizations for neonatal infectious diseases included respiratory tract infections, sepsis, gastric intestinal infections and dermatologic infections in our study. Q: quarter

diseases in different quarters of the COVID-19 cohort significantly declined as compared with those forecasted ( $300.25 \pm 57.33$ vs $546.64 \pm 100.43, P=0.006)$.

The proportions of hospitalizations for neonatal infectious diseases to the total hospitalizations of neonates in different quarters of 2020 were forecasted by Winters' additive model with the data in the pre-COVID-19 cohort. As shown in Fig. 5b, the proportions of hospitalizations for neonatal infectious diseases to the total hospitalizations of neonates in different quarters of the COVID-19 cohort significantly decreased as compared with those forecasted: the first quarter $(0.34$ vs 0.40 , $P=0.002)$, the second quarter $(0.24$ vs $0.30, P=0.001)$, the third quarter $(0.24$ vs $0.28, P=0.024)$, and the fourth quarter $(0.29$ vs $0.35, P=0.003)$.

\section{Discussion}

This is a cross-sectional study on infectious disease hospitalizations of neonates during 2015-2020, focusing on the impact of COVID-19 pandemic on infectious disease hospitalizations. Although perinatological interventions and breakthroughs in neonatology have led to advances in reduction of neonatal morbidity and mortality $[13,14]$, infectious diseases remain to be the persistent threat of neonatal health, corresponding to a mean neonatal infectious disease hospitalization rate of over one third in our study. In the interest of public health, a lot of preventive measures have been introduced to curb the COVID-19 pandemic since January 2020. The neonates with infectious diseases have been dramatically impacted by these preventive measures. Thus, it is important to monitor infectious diseases of neonates to better understand their effect on the health care system and neonatal health.

Among neonatal factors that predispose neonates to infections, the status of the innate and adaptive immune response can play a significant role [15]. Early onset infectious diseases that compromise early newborns are mainly due to infections occurring during the antenatal or intrapartum period [16], which is usually described as "vertical transmission". And late onset infectious diseases that compromise late newborns result from pathogens acquired from the environment after birth [17], which is also known as "horizontal transmission". The significant decreases in the number of infectious disease hospitalizations in both the early and late newborn suggest the COVID-19 pandemic educational/governmental mitigation strategies can effectively reduce both vertical and horizontal transmissions of infectious disease in neonates, and the results could provide us with some beneficial enlightenment for prevention of community- and hospital-acquired infections of neonates. The COVID-19 pandemic educational/governmental mitigation strategies are certainly effective in the prevention of infectious diseases with horizontal transmissions, but the exact mechanism by which the COVID-19 mitigation strategies protect the neonates from vertically transmitted infections is unclear, which needs to be further investigated.

The respiratory tract infection was the most commonly listed cause of infection among infectious disease hospitalizations of neonates, and sepsis, gastric intestinal infections and dermatologic infections were listed as the other three main causes in infectious disease hospitalizations of neonates. These results are consistent with data 


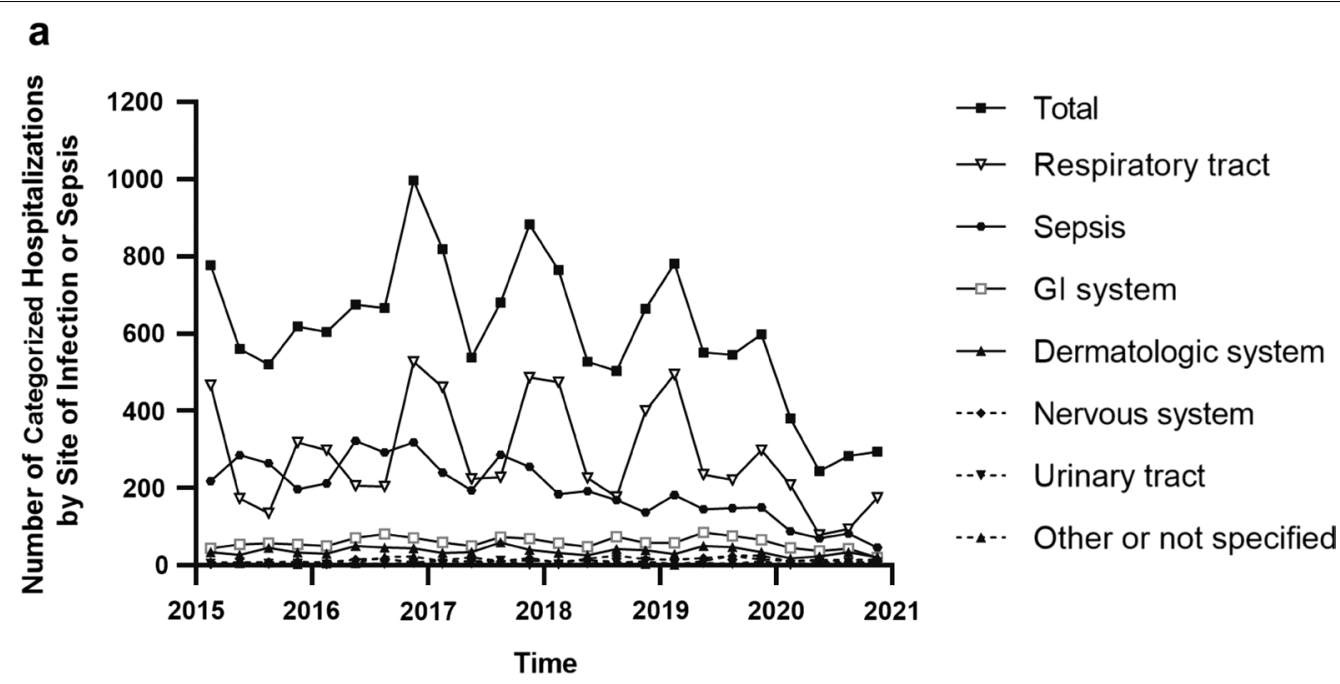

b

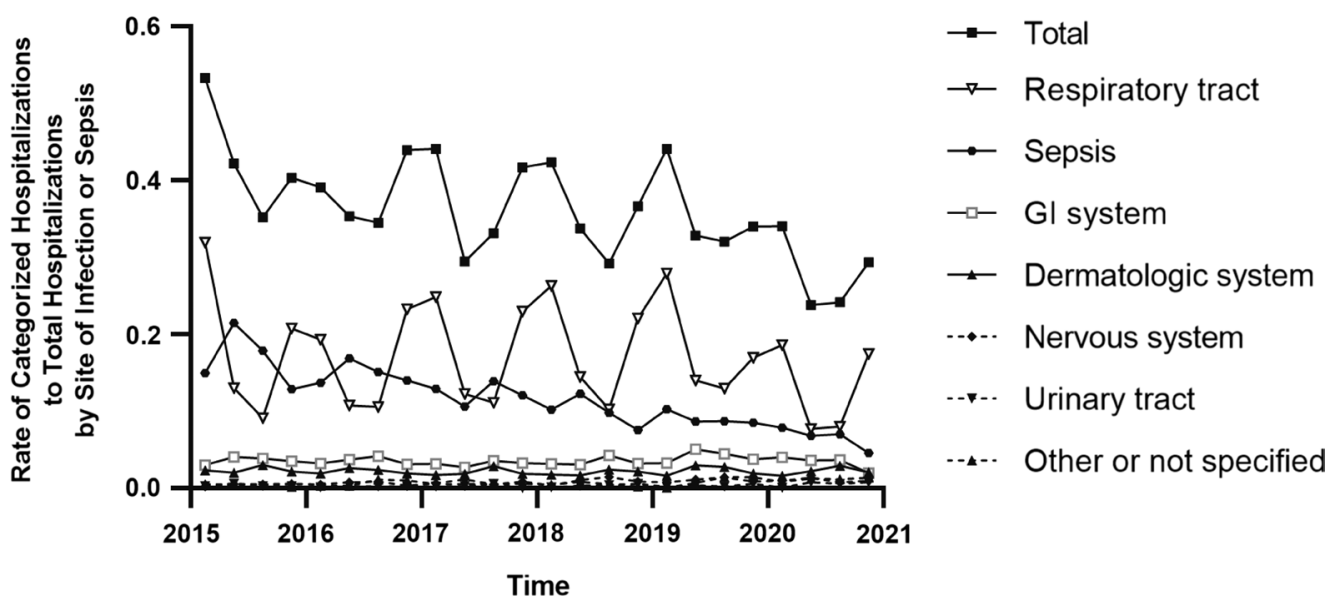

Fig. 4 Infectious disease hospitalizations of neonates by the seasonality. a The number of categorized infectious disease hospitalizations of neonates. $\mathbf{b}$ The rate of categorized infectious disease hospitalizations of neonates

documenting that the lower respiratory tract infection had the highest mean annual age-adjusted rate among infectious disease hospitalizations in the United States during 2001 to 2014 [18].

The prevalence of the infectious disease hospitalizations of neonates had significant seasonality with a high rate in winter and spring, especially respiratory tract infection hospitalizations of neonates. These results suggest considerable infectious disease hospitalizations of neonates that are likely to be due to climate factors that could alter the epidemiology of climate sensitive pathogens and host immune response. The association between climate factors and infectious disease is very complex and influenced by many factors such as socioeconomic status, viral prevalence, temperature, precipitation, relative humidity, and an increase in the level of air pollutants [19-21]. Further investigation into the relation between climate factors and neonatal infectious diseases is warranted.

We observed the numbers as well as the proportions of hospitalizations for neonatal infectious diseases in different quarters of 2020 dramatically declined. The main factor that drives such decreases in infectious disease hospitalizations of neonates is the impact of the COVID-19 pandemic. A previously unknown coronavirus was first discovered in samples from patients with pneumonia in December 2019, which triggered a global pandemic. The COVID-19 pandemic and its mitigation strategies have caused an unprecedented impact on all aspects of social and economic life, especially on human health and healthcare systems worldwide. Compliance of people with preventive measures and policies such as social distancing, face masking, sanitation, and isolation has played an important role in mitigating the pandemic 


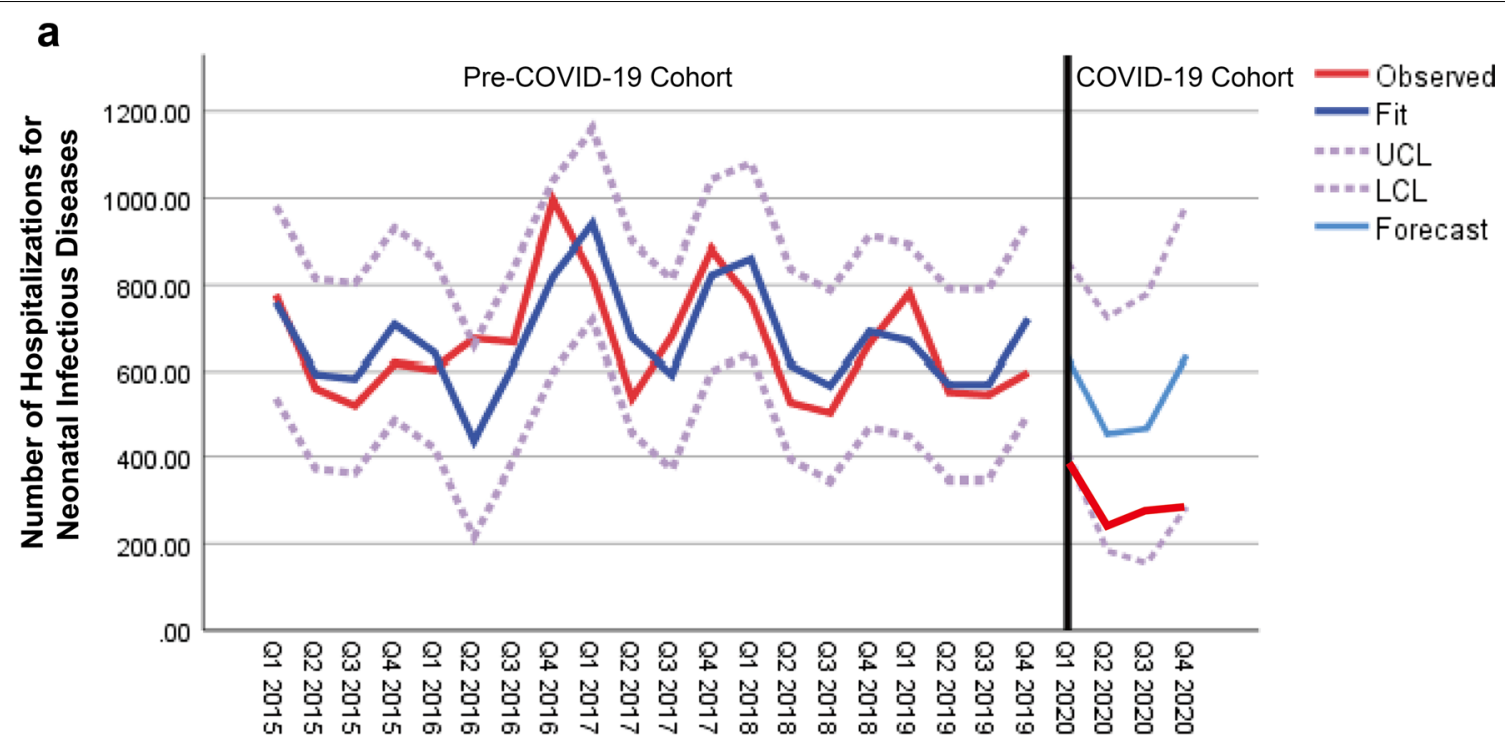

Time

b

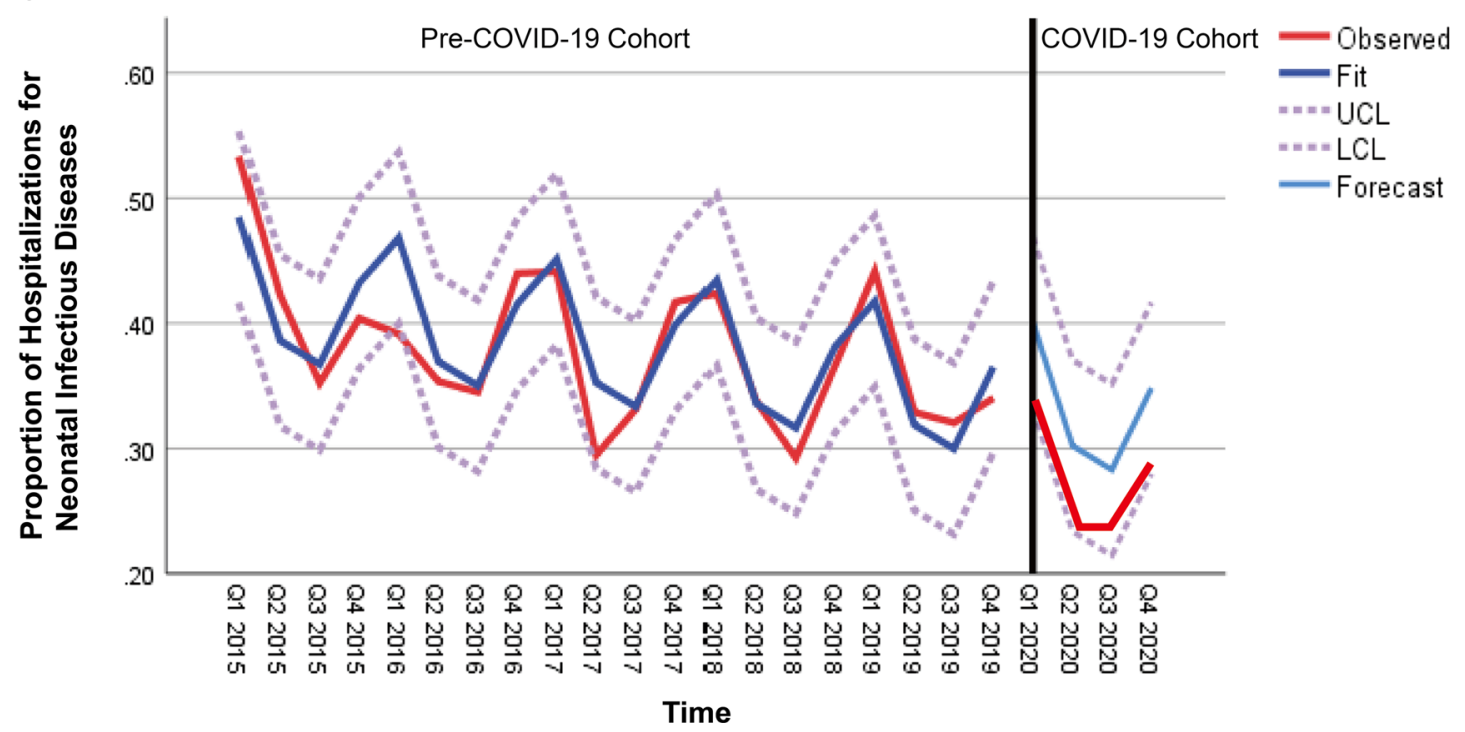

Fig. 5 Infectious disease hospitalizations of neonates by the impact of the COVID-19 pandemic. Both the observed numbers (a) and proportions (b) of hospitalizations for neonatal infectious diseases in different quarters of the COVID-19 cohort significantly declined as compared with those forecasted. UCL upper confidence limit, LCL lower confidence limit, Q quarter

as well as decreasing the prevalence of infectious disease of neonates. The mitigation strategies and preventive measures which are very effective in fighting the pandemic have contributed a lot in reducing the incidence of infectious disease of neonates. It is worth mentioning that public psychological factors should be considered. Although there have been no government recommendations or hospital policies to limit hospitalization admission or outpatient treatment during the COVID-19 pandemic, a few people might have avoided hospital visits and hospitalizations because they were worried about being infected by COVID-19 while going to hospital. Fortunately, there has been no significant increase in overall neonatal mortality in our province during the COVID19 pandemic according to the recent public data. 


\section{Study limitations}

This study had a few limitations. First, epidemiological data on hospital admissions for infectious diseases of neonates, the basis for this study, are subject to bias and other selective pressures that might affect principal diagnosis, such as reluctance to seek timely medical advice and treatment for fear of contracting the novel coronavirus in health care settings. Second, the study is a singlecenter based cross-sectional study with a relatively small sample. Third, we did not collect data on the pathogen type of the hospitalized newborn infants with infectious diseases. Finally, there were only inpatient data included in our study and no outpatient data.

\section{Conclusion}

Despite the outbreak of the COVID-19 pandemic, the leading causes of hospitalizations for neonatal infectious diseases remain being respiratory tract infections, sepsis, gastric intestinal infections and dermatologic infections in our study. The numbers as well as the overall rates of hospitalizations for neonatal infectious diseases in different quarters of 2020 dramatically declined because of the impact of the COVID-19 pandemic and its mitigation measures.

\section{Abbreviations \\ COVID-19: Coronavirus disease 2019; ICD-10: International Classification of Diseases, version 10.}

\section{Supplementary Information}

The online version contains supplementary material available at https://doi. org/10.1186/s12879-022-07211-x.

Additional file 1. Distribution of the number of infectious disease hospitalizations of neonates, the Children's Hospital, Zhejiang University School of Medicine, 2015-2020.

\section{Acknowledgements}

We would like to thank Litao Zhou (The Children's Hospital, Zhejiang University School of Medicine, National Clinical Research Center for Child Health) for helping us with data collection.

\section{Authors' contributions}

JP and TY conceptualized and designed the study, drafted the initial manuscript, and reviewed and revised the manuscript. CZ, YS, and WW designed the data collection instruments, collected data, carried out the initial analyses, and reviewed and revised the manuscript. LC supervised data collection, and critically reviewed the manuscript for important intellectual content. All authors approved the final manuscript as submitted and agreed to be accountable for all aspects of the work. All authors read and approved the final manuscript.

\section{Funding}

No funding was secured for this study.

\section{Availability of data and materials}

The used or analyzed data during the current study is available from the corresponding author on reasonable request.

\section{Declarations}

Ethics approval and consent to participate

The study was carried out in accordance with the principles of the Declaration of Helsinki and approved by the Ethics Committee of the Children's Hospital, Zhejiang University School of Medicine (IRB/EC Ref No. 2021-IRB-166). The need for individual informed consent was waived by the Ethics Committee of the Children's Hospital, Zhejiang University School of Medicine because of the retrospective nature and anonymity of the study.

\section{Consent for publication}

Not applicable.

\section{Competing interests}

The authors declare that they have no competing interests.

Received: 14 November 2021 Accepted: 25 February 2022

Published online: 02 March 2022

\section{References}

1. Nanduri SA, Petit S, Smelser C, Apostol M, Alden NB, Harrison LH, Lynfield R, Vagnone PS, Burzlaff K, Spina NL, et al. Epidemiology of invasive earlyonset and late-onset group B streptococcal disease in the United States, 2006 to 2015: multistate laboratory and population-based surveillance. JAMA Pediatr. 2019;173(3):224-33.

2. Schrag SJ, Farley MM, Petit S, Reingold A, Weston EJ, Pondo T, Hudson Jain J, Lynfield R. Epidemiology of invasive early-onset neonatal sepsis, 2005 to 2014. Pediatrics. 2016;138(6):e20162013.

3. Giannoni E, Agyeman PKA, Stocker M, Posfay-Barbe KM, Heininger U, Spycher BD, Bernhard-Stirnemann S, Niederer-Loher A, Kahlert CR, Donas A, et al. Neonatal sepsis of early onset, and hospital-acquired and community-acquired late onset: a prospective population-based cohort study. J Pediatr. 2018;201:106-14.

4. Yorita KL, Holman RC, Sejvar JJ, Steiner CA, Schonberger LB. Infectious disease hospitalizations among infants in the United States. Pediatrics. 2008; 121(2):244-52.

5. Zhu N, Zhang D, Wang W, Li X, Yang B, Song J, Zhao X, Huang B, Shi W, Lu $R$, et al. A novel coronavirus from patients with pneumonia in China, 2019. N Engl J Med. 2020;382(8):727-33.

6. Barbuddhe SB, Rawool DB, Gaonkar PP, Vergis J, Dhama K, Malik SS. Global scenario, public health concerns and mitigation strategies to counter current ongoing SARS-CoV-2/COVID-19 pandemic. Hum Vaccin Immunother. 2020;16(12):3023-33.

7. Sharma A, Tiwari S, Deb MK, Marty JL. Severe acute respiratory syndrome coronavirus-2 (SARS-CoV-2): a global pandemic and treatment strategies. Int J Antimicrob Agents. 2020;56(2):106054.

8. Levin AT, Hanage WP, Owusu-Boaitey N, Cochran KB, Walsh SP, Meyerowitz-Katz G. Assessing the age specificity of infection fatality rates for COVID-19: systematic review, meta-analysis, and public policy implications. Eur J Epidemiol. 2020;35(12):1123-38.

9. Liu W, Yue XG, Tchounwou PB. Response to the COVID-19 epidemic: The Chinese experience and implications for other countries. Int J Environ Res Public Health. 2020;17(7):2304.

10. Kraemer MUG, Yang CH, Gutierrez B, Wu CH, Klein B, Pigott DM, C-DWG Open, du Plessis L, Faria NR, Li R, et al. The effect of human mobility and control measures on the COVID-19 epidemic in China. Science. 2020;368(6490):493-7.

11. Zhu FC, Guan XH, Li YH, Huang JY, Jiang T, Hou LH, Li JX, Yang BF, Wang L, Wang WJ, et al. Immunogenicity and safety of a recombinant adenovirus type-5-vectored COVID-19 vaccine in healthy adults aged 18 years or older: a randomised, double-blind, placebo-controlled, phase 2 trial. Lancet. 2020;396(10249):479-88.

12. Zhou $X$, Song Y, Jiang H, Wang Q, Qu Z, Zhou X, Jit M, Hou Z, Lin L. Comparison of public responses to containment measures during the initial outbreak and resurgence of COVID-19 in China: infodemiology study. J Med Internet Res. 2021;23(4):e26518. 
13. Polin RA, Carlo WA, Committee on F, Newborn, American Academy of P. Surfactant replacement therapy for preterm and term neonates with respiratory distress. Pediatrics. 2014;133(1):156-63.

14. Russell NJ, Seale AC, O'Sullivan C, Le Doare K, Heath PT, Lawn JE, Bartlett L, Cutland C, Gravett M, Ip M, et al. Risk of early-onset neonatal group b streptococcal disease with maternal colonization worldwide: systematic review and meta-analyses. Clin Infect Dis. 2017;65(suppl_2):S152-9.

15. Basha S, Surendran N, Pichichero M. Immune responses in neonates. Expert Rev Clin Immunol. 2014;10(9):1171-84.

16. Simonsen KA, Anderson-Berry AL, Delair SF, Davies HD. Early-onset neonatal sepsis. Clin Microbiol Rev. 2014;27(1):21-47.

17. Dong Y, Speer CP. Late-onset neonatal sepsis: recent developments. Arch Dis Child Fetal Neonatal Ed. 2015;100(3):F257-263.

18. Kennedy JL, Haberling DL, Huang CC, Lessa FC, Lucero DE, Daskalakis DC, Vora NM. Infectious Disease Hospitalizations: United States, 2001 to 2014. Chest. 2019;156(2):255-68.

19. Hossain MZ, Bambrick H, Wraith D, Tong S, Khan AF, Hore SK, Hu W. Sociodemographic, climatic variability and lower respiratory tract infections: a systematic literature review. Int J Biometeorol. 2019;63(2):209-19.

20. Mirsaeidi M, Motahari H, Taghizadeh Khamesi M, Sharifi A, Campos M, Schraufnagel DE. Climate change and respiratory infections. Ann Am Thorac Soc. 2016;13(8):1223-30.

21. Stern G, Latzin P, Roosli M, Fuchs O, Proietti E, Kuehni C, Frey U. A prospective study of the impact of air pollution on respiratory symptoms and infections in infants. Am J Respir Crit Care Med. 2013;187(12):1341-8.

\section{Publisher's Note}

Springer Nature remains neutral with regard to jurisdictional claims in published maps and institutional affiliations.

Ready to submit your research? Choose BMC and benefit from:

- fast, convenient online submission

- thorough peer review by experienced researchers in your field

- rapid publication on acceptance

- support for research data, including large and complex data types

- gold Open Access which fosters wider collaboration and increased citations

- maximum visibility for your research: over 100M website views per year

At BMC, research is always in progress.

Learn more biomedcentral.com/submissions 